\title{
Spotlight on perampanel in the management of seizures: design, development and an update on place in therapy
}

This article was published in the following Dove Press journal:

Drug Design, Development and Therapy

4 October 2017

Number of times this article has been viewed

\author{
Michele A Faulkner ${ }^{1,2}$ \\ 'Department of Pharmacy Practice, \\ ${ }^{2}$ Department of Neurology, Creighton \\ University Schools of Pharmacy and \\ Medicine, Omaha, NE, USA
}

Purpose: Perampanel is a first-in-class antiepileptic medication approved for the treatment of partial (focal) seizures, and as adjunctive treatment for primarily generalized tonic-clonic seizures. The pharmacology, efficacy data, adverse-effect profile, pharmacokinetics and place in therapy are reviewed.

Summary: Perampanel is indicated for use in patients with epilepsy who are 12 years of age or older. It is the first medication designed specifically to be a non-competitive antagonist at postsynaptic $\alpha$-amino-3-hydroxyl-5-methyl-4-isoxazole-propionate receptors. Efficacy in refractory seizures has been established, and ongoing efficacy demonstrated by post-marketing data. The drug is completely absorbed, and exhibits a half-life that allows for once-daily administration in doses up to $12 \mathrm{mg} /$ day. Drug interactions are minimal, but increased doses may be necessary when given with strong inducers of cytochrome P450 enzymes, including when perampanel is co-administered with other antiepileptics that exhibit this property. The most common adverse effects noted in both clinical trials and post-marketing are dizziness and somnolence. Psychiatric and behavioral adverse events have been documented in both adult and pediatric patients, including those with no corresponding diagnostic history.

Conclusion: Perampanel is a novel adjunctive antiepileptic medication that is an effective option for adolescents and adults with partial seizures, and primarily generalized tonic-clonic seizures uncontrolled with other medications.

Keywords: perampanel, fycompa, focal seizures, partial seizures, generalized seizures

\section{Introduction}

Approximately 50 million individuals worldwide have epilepsy. ${ }^{1}$ Partial (focal) seizures comprise roughly $60 \%$ of epileptic seizures. To date, no medication approved as a monotherapeutic agent for the treatment of partial seizures has emerged as substantially more efficacious than any other. The typical efficacy rate for these medications is between $20 \%$ and $50 \%{ }^{2}$ As such, adjunctive therapy is frequently necessary. Additionally, while generalized seizures are considered easier to control than partial seizures, many patients remain refractory to current treatment modalities. Few medications with novel mechanisms have been approved in recent years for the purpose of targeting generalized seizures. ${ }^{3}$

Drugs approved by the US Food and Drug Administration (FDA) for the initial treatment of partial seizures include older agents such as phenytoin, carbamazepine and phenobarbital (all strong hepatic enzyme inducers), as well as valproic acid (and enzyme-inhibiting medication). Newer medications approved for partial seizure monotherapy include oxcarbazepine, topiramate, felbamate, lacosamide, eslicarbazepine
Correspondence: Michele A Faulkner Departments of Pharmacy Practice and Neurology, Creighton University School of Pharmacy, 2500 California Plaza,

Omaha, NE 69178, USA

$\mathrm{Tel}+\mathrm{I} 4022804197$

Fax +I 4022803320

Email faulkner@creighton.edu 
and lamotrigine (after conversion from a failed monotherapeutic alternative). A number of other medications have been approved for adjunctive treatment of partial seizures (felbamate, gabapentin, levetiracetam, pregabalin, tiagabine, vigabatrin and zonisamide). Several of these newer medications have novel mechanisms of action. While rational polytherapy (employing drugs with different mechanisms of action) would seem a logical method for improving control of seizures that are refractory to a single agent, this has not been explicitly demonstrated. ${ }^{4}$ Still, the discovery of antiepileptics with novel pharmacologic properties is of significant interest.

\section{Perampanel}

\section{Pharmacology}

Perampanel (Fycompa; Eisai Inc, Woodcliff Lake, NJ, USA) is a novel antiepileptic drug that has affinity for excitatory post-synaptic $\alpha$-amino-3-hydroxy-5-methyl-4-isoxazolepropionate (AMPA) glutamate receptors where it acts as a selective antagonist. ${ }^{5}$ It binds to a separate site from glutamate, but as a non-competitive inhibitor, it prevents the normal excitatory physiologic response to glutamate from occurring while still allowing it to bind to the receptor. ${ }^{6}$ While there are other drugs that act at glutamate receptors (felbamate at the N-methyl-D-aspartate (NMDA) receptor, and topiramate at the kainite receptor), perampanel is the first medication to be specifically designed with glutamate inhibition as its primary mechanism of action. ${ }^{7,8}$

The rationale for targeting glutamate receptors stems from the fact that extracellular glutamate concentrations in the hippocampus become elevated prior to and during seizure activity. ${ }^{9}$ As glutamate is the primary excitatory neurotransmitter in the brain, associated receptors have been deemed a logical target for antiepileptic activity. Previous attempts to target glutamate via NMDA receptors have met with disappointing results owing to associated neuropsychiatric side effects. ${ }^{10,11}$ As such, targeting other glutamate receptors, such as AMPA receptors, became a logical alternative. Of note, AMPA receptors are the foremost mediators of excitatory neurotransmission, and are the most frequently encountered receptor type in the central nervous system. ${ }^{5,11}$

AMPA receptors are ligand-gated cation channels with 4 separate agonist binding sites. ${ }^{5}$ The speed of channel opening is directly proportional to the number of concurrently occupied sites. ${ }^{12,13}$ Because the channels open and close quickly, it is hypothesized that the AMPA receptors play a large role in facilitating most of the fast excitatory transmission in the central nervous system. Perampanel was designed specifically to inhibit this transmission in the hope that the propagation of seizure activity would be prevented.

\section{Pharmacokinetics}

The pharmacokinetic profile of perampanel is a linear, 1-compartment model with first-order elimination. ${ }^{14}$ Absorption is rapid with maximum concentrations achieved between 0.5 and 2.5 hours after ingestion. ${ }^{15}$ Bioavailability approaches nearly $100 \%$, and food does not have an effect, though the time to reach the maximum concentration may be delayed by ingesting a meal at the time of dosing. ${ }^{16}$ The half-life of perampanel is long, and typically falls between 52 and 129 hours for a single dose. After 2 weeks of dosing, which is approximately the amount of time it takes to reach steady state, the half-life is typically between 66 and 90 hours. ${ }^{17}$ The drug is highly bound (95\%), but significant binding interactions have not been identified. Oxidation via CYP3A4 and CYP3A5 followed by glucuronidation is the primary metabolic pathway for perampanel. ${ }^{18}$ Due to a decrease of nearly $50 \%$ in patients with mild or moderate hepatic disease, it is recommended that doses be adjusted for individuals with a Child-Pugh score of A or B. ${ }^{18}$ Data are lacking in those with severe liver disease, and subsequently it is recommended that the drug be avoided in individuals with this diagnosis. Likewise, a lack of data in persons with severe renal disease or those undergoing hemodialysis has led to a recommendation of perampanel avoidance. ${ }^{18}$

\section{Efficacy}

Three Phase III trials demonstrated the efficacy of perampanel as adjunctive therapy for the treatment of partial seizures, and led to its approval for this indication. All 3 of the trials had the same basic design (randomized, double-blind, placebo controlled trials, including subjects of at least 12 years of age who were taking no more than 3 additional antiepileptic agents for the control of partial seizures with or without secondary generalization). ${ }^{19-21}$ In each of the trials, titration of drug occurred over a 6-week time period ( $2 \mathrm{mg}$ per week to the target dose outlined in the design of the study). That was followed by a 13 -week maintenance phase. The primary outcome measures were the change in seizure frequency per 28 days during the entire trial compared with baseline, and the number of subjects experiencing a minimum decrease in seizure frequency of at least 50\% (known as the responder rate or RR). Outcomes from the Phase III trials of perampanel for the adjunctive treatment of partial seizures are summarized in Table 1. Of note, data from study 304 demonstrated no significant difference in the RR when the entire group of 
Table I Summary of Phase III efficacy trials of perampanel as adjunctive therapy for partial seizures

\begin{tabular}{|c|c|c|c|c|}
\hline Study & $\begin{array}{l}\text { Number } \\
\text { of subjects }\end{array}$ & $\begin{array}{l}\text { Median percent change in } \\
\text { seizure frequency from baseline } \\
\text { (significance vs placebo) }\end{array}$ & Responder rate & $\begin{array}{l}\text { Seizure } \\
\text { freedom }\end{array}$ \\
\hline $304^{19}$ & 387 & $\begin{array}{l}\text { Placebo }=21 \%, 8 \mathrm{mg} / \text { day }=26.3 \% \\
(p=0.026) \\
12 \mathrm{mg} / \text { day }=34.5 \% \\
(p=0.016)\end{array}$ & $\begin{array}{l}\text { Placebo }=26.4 \%, \\
8 \mathrm{mg} / \text { day }=37.6 \% \\
(p=0.076),{ }^{\mathrm{a}} \\
12 \mathrm{mg} / \text { day }=36.1 \% \\
(p=0.091)^{\mathrm{a}}\end{array}$ & $\begin{array}{l}\text { Placebo }=0 \%, \\
8 \mathrm{mg} / \text { day }=2.2 \%, \\
12 \mathrm{mg} / \text { day }=1.5 \%\end{array}$ \\
\hline $305^{20}$ & 386 & $\begin{array}{l}\text { Placebo }=9.7 \% \\
8 \mathrm{mg} / \text { day }=30.5 \% \\
(p<0.00 \mathrm{I}) \\
12 \mathrm{mg} / \text { day }=17.6 \% \\
(p=0.0 \mathrm{II})\end{array}$ & $\begin{array}{l}\text { Placebo }=\mid 4.7 \%, \\
8 \mathrm{mg} / \text { day }=33.3 \% \\
(p=0.002), \\
\mathrm{I} 2 \mathrm{mg} / \text { day }=33.9 \% \\
(p<0.00 \mathrm{I})\end{array}$ & $\begin{array}{l}\text { Placebo }=1.5 \%, \\
8 \mathrm{mg} / \text { day }=2.3 \%, \\
12 \mathrm{mg} / \text { day }=5 \%\end{array}$ \\
\hline $306^{21}$ & 705 & $\begin{array}{l}P \text { lacebo }=10.7 \%, \\
2 \mathrm{mg} / \mathrm{day}=13.6 \% \\
(\mathrm{NS}), \\
4 \mathrm{mg} / \mathrm{day}=23.3 \% \\
(p=0.0026), \\
8 \mathrm{mg} / \mathrm{day}=30.8 \% \\
(p<0.000 \mathrm{I})\end{array}$ & $\begin{array}{l}\text { Placebo }=17.9 \%, \\
2 \mathrm{mg} / \text { day }=20.6 \% \\
(\mathrm{NS}), \\
4 \mathrm{mg} / \mathrm{day}=28.5 \% \\
(p=0.0132), \\
8 \mathrm{mg} / \mathrm{day}=34.9 \% \\
(p=0.0003)\end{array}$ & $\begin{array}{l}\text { Placebo }=1.2 \%, \\
2 \mathrm{mg} / \text { day }=1.9 \%, \\
4 \mathrm{mg} / \text { day }=4.4 \%, \\
8 \mathrm{mg} / \text { day }=4.8 \%\end{array}$ \\
\hline
\end{tabular}

Note: a 8 and $12 \mathrm{mg} /$ day were both significant vs placebo $(p<0.005)$ when considering North American data only.

Abbreviation: NS, non significant.

study subjects was evaluated. ${ }^{19}$ However, individuals enrolled in North American centers did have an RR that reached significance ( $p<0.05$ for both doses). It has been theorized that selection of participants or specific behaviors exhibited by investigators in the Central and South American trial centers may have played a role in creating this discrepancy, but no specific explanation has been disclosed.

Results from an open-label extension trial that enrolled patients from all 3 of the Phase III trials undertaken in patients with drug-resistant partial seizures (study 307) bear out the efficacy of perampanel over time. ${ }^{22}$ After a 16-week blinded conversion period during which all patients previously randomized to placebo were switched to perampanel (titrated by $2 \mathrm{mg}$ increments on a bi-weekly basis) to a maximum of $12 \mathrm{mg} /$ day, reductions in seizure frequency were measured. Those previously randomized to perampanel achieved a rate of seizure reduction of $41.4 \%$ vs a decrease of $44.3 \%$ in the newly converted subjects. Over the total 52-week maintenance period, reductions were $55 \%$ and $53.9 \%$ in the former placebo group $(n=209)$ and those who originally received active drug $(\mathrm{n}=485)$, respectively. Most subjects reported adverse events during the study, but rates did not differ between the 2 groups $(90.7 \%$ in former placebo recipients vs $91.5 \%$ for perampanel users). The study withdrawal rate in those who had always been taking perampanel was $14.3 \%$, which was lower than the rate in those switching over from placebo (19.8\%). This can likely be explained by the fact that patients needed to cease therapy in the always-perampanel users did so during the original Phase III studies. As seizure control was maintained in patients who received perampanel in the double-blind trials, this extension study would seem to demonstrate that efficacy is maintained over the long term. ${ }^{19}$

The number of additional antiepileptic medications taken by study subjects was a predictor of efficacy in the Phase III trials evaluating efficacy in partial seizures. ${ }^{23}$ For those individuals who were using a single additional antiepileptic agent, the median percent decrease in seizures per 28 days was significantly higher compared with individuals who were using 3 additional medications $(p<0.02)$. However, no difference was noted between users of 1 vs 2 additional drugs, or 2 vs 3 ( $p=0.06$ and $p>0.2$, respectively). The responder rate was also higher in those who only needed 1 additional medication $(p<0.02)$. No difference was noted in the rate of seizure freedom.

Post-marketing data suggest that perampanel is efficacious for patients with refractory epilepsy outside of the setting of clinical trials. A Spanish, multi-center, retrospective study that spanned 1-year evaluated outcomes data $(n=281)$ in patients with partial seizures with or without secondary generalization. ${ }^{24}$ The overall decrease in seizure frequency was $77.4 \%$ in patients with simple partial seizures, and $58.1 \%$ in those with complex partial seizures. The corresponding RR was $40.2 \%$. Seizure freedom occurred in $10.3 \%$ of perampanel users. Individuals who had tried fewer than 6 other antiepileptic medications were more likely to experience 
seizure freedom compared with those whose seizures had proven refractory to a higher number of drugs ( $p=0.004)$. The RR was also more robust in this group of patients $(p=0.027)$. Of note, this study included patients receiving more than 3 antiepileptic medications concurrently with perampanel, and thus, included a population that would have been excluded from the Phase III clinical efficacy trials.

In July, 2017, perampanel received FDA approval for use as monotherapy in patients with partial seizures with or without secondary generalization. ${ }^{25}$ This marks the first drug approval by the FDA using a new regulatory pathway that analyzes extrapolated data to determine if a medication should be approved for monotherapy without a prospective trial to gather data on the drug as a single agent. Data extrapolated from the open-label extension studies was utilized to fulfill this purpose. ${ }^{26}$ Seven subjects transitioned from using perampanel as adjunctive therapy to utilizing it as the sole agent to control seizures. Of those, 6 had outcome data available for assessment. All demonstrated a decrease in seizure frequency of at least $50 \%$ at the time of their last follow up. There were 5 treatment-emergent adverse events in this group of subjects, one of which was considered serious (colitis). However, colitis is not a recognized adverse event associated with perampanel use. In addition, data from a retrospective, noninterventional, multi-center (19 locations) studies provided information informing the decision to approve perampanel as monotherapy. ${ }^{27}$ The primary outcome was the retention rate of 60 patients who received perampanel between January 1, 2013, and March 1, 2016, with evaluations taking place at 3, 6, 12, 18 and 24 months. Patients were either converted to monotherapy $(n=51)$, or they began perampanel as their sole antiepileptic treatment upon therapy initiation. At the end of the study, $68.3 \%$ of patients were still receiving perampanel monotherapy. Fourteen patients were taking perampanel in conjunction with at least one other antiepileptic medication. A total of 40 patients were included in the efficacy analysis. The median decrease in seizure frequency per 28 days during the first 3 months of monotherapy was $81.7 \%$ $(n=40)$. During the final 3 months (including only patients who had been using the drug in this manner for a minimum of 6 months, $n=22$ ), the median decrease was $100 \%$. Seizure freedom for a minimum of 3 months at any point during the study was achieved by $55 \%$ of patients. The RR for the last 3 months of the study was $90.9 \%$, and $81.8 \%$ of patients had a decrease in seizure frequency of at least $70 \%$.

Since the original approval of perampanel for the adjunctive treatment of partial seizures was obtained, additional data was presented to the FDA leading to approval of the drug for the adjunctive treatment of primarily generalized tonic-clonic seizures in June of 2015. In a study that enrolled subjects of at least 12 years of age, who were using between 1 and 3 additional antiepileptic medications, had a diagnosis of primary generalized tonic-clonic seizures and idiopathic generalized epilepsy, perampanel was found to be effective for the treatment of refractory seizures. ${ }^{28}$ The target maximum dose for the study was $8 \mathrm{mg}$ /day (achieved by $80.2 \%$ ), or the highest dose tolerated by the subject. Titration to the target dose occurred over 4 weeks. A 13-week maintenance phase followed. Efficacy was measured by the primary endpoint of percent decrease in seizure frequency per 28 days, and the secondary endpoint, the RR. Eighty-one individuals were randomized to each group. For those receiving perampanel, the decrease in seizure frequency was significant compared with placebo (38.4\% vs $76.5 \%, p<0.001)$. The same held true for the RR $(64.2 \%$ and $39.5 \%$, respectively, for the perampanel and placebo groups, $p=0.0019)$. Seizure freedom was noted in $30.9 \%$ of those on active medication compared with $12.3 \%$ of those receiving placebo. Taking into consideration all seizure types experienced by study subjects, the perampanel group achieved the primary endpoint significantly more often than did the placebo group (43.4\% vs $22.9 \%, p=0.0018$ ).

Efficacy data has also emerged for several special populations. A retrospective study evaluating the efficacy of the drug in patients with learning disabilities and/or pre-existing psychiatric comorbidities who also had refractory epilepsy was recently published. ${ }^{29}$ Specifically, of the 101 patients studied, 37.6\% had learning disabilities, and 49.5\% were diagnosed with a psychiatric disorder (not otherwise specified). After perampanel therapy was initiated, $41.6 \%$ of patients achieved a $50 \%$ or greater drop in seizure frequency, and 7 became free of seizure activity. Another retrospective study was completed in the Netherlands and comprised a population of patients with intellectual disabilities $(n=62){ }^{30}$ Mean age of seizure onset was 6 years. The average number of antiepileptics being given was 4 indicating a population with highly refractory seizures. While none of the patients became free of seizures, over half experienced a decrease in seizure activity with the addition of perampanel.

Individuals considered elderly (age at least 65 years) who were enrolled in the original Phase III trials had been evaluated separately from the total population. ${ }^{31}$ Despite being a rapidly growing segment of the epilepsy population, they remained underrepresented in clinical trials. The underlying etiology of seizures in the 28 subjects evaluated was stroke for $7.1 \%$ in comparison with the remainder of the study population where stroke was the underlying cause of seizure 
activity for $1.1 \%$. The lower doses, 2 and $4 \mathrm{mg} /$ day, were not taken by enough patients to make the data meaningful. For those elderly individuals receiving 8 or $12 \mathrm{mg}$ /day, $16.9 \%$ and $12.5 \%$ met the primary endpoint, respectively. This was in comparison with $6.8 \%$ for elderly subjects receiving placebo. The RR in the $8 \mathrm{mg}$ /day group was $22.2 \%$, and in the $12 \mathrm{mg}$ /day group was $42.9 \%$ vs $25 \%$ in the placebo group. There appeared to be no loss of efficacy in older patients using perampanel.

\section{Safety and tolerability}

Two Phase II trials, studies 206 and 208, demonstrated equal rates of adverse events in subjects receiving active drug and those who took placebo. ${ }^{32}$ Doses ranged from $4 \mathrm{mg}$ /day (all subjects in study 206) and $12 \mathrm{mg} /$ day (the max dose for study 208). Six individuals, 3 in each study group, withdrew from study 206 due to adverse events. For study 208, there were 2 withdrawals from the perampanel group, and 1 from the placebo group. Adverse event rates were $62.7 \%$ and $66.7 \%$ (study 206), and $80 \%$ and $84.2 \%$ (study 208) for the placebo and perampanel groups, respectively. In both the Phase II trials, dizziness and somnolence were reported by $>10 \%$ of subjects using perampanel. It should be noted that these are well-known adverse events among the antiepileptic drug class, and thus it is not possible to determine if perampanel was the causative medication. No laboratory abnormalities or deaths were noted in either study. ${ }^{32}$

The 4 trials evaluating perampanel for partial seizures (studies 304-307) demonstrated an overall adverse event rate of $87.4 \%{ }^{19-21,33}$ The majority were attributed to treatment, with $9.2 \%$ considered unrelated. For the most part, dose-response relationships were evident. Two-thirds of the reactions were mild or moderate in intensity, which is consistent with the data that emerged from the Phase II trials. Dizziness, irritability and aggression were each responsible for discontinuation by at least $1 \%$ of the study population. Overall, $13.2 \%$ of subjects withdrew from the studies due to adverse events. For individuals receiving the highest studied dose (12 mg/day), $\sim 10 \%$ experienced a fall during treatment. Dose reductions were necessary for $36.1 \%$ of subjects during the titration phase, and another $3.3 \%$ required interruption in therapy. Dizziness was the most frequent reason for both ( $>20 \%$ of instances), while somnolence, ataxia, fatigue, headache and gait disturbance were all responsible for treatment alterations in at least $2 \%$ of study participants. No patient experienced sudden unexpected death in epilepsy (SUDEP) during the blinded trials, though there was a single case reported in the extension trial. ${ }^{33}$ Five subjects reported thoughts of suicide with perampanel doses of $2 \mathrm{mg}$ /day ( 2 subjects) and $8 \mathrm{mg} /$ day ( 2 subjects), and 1 patient taking placebo. Weight gain ( $>7 \%$ from baseline) was noted in between $11.6 \%$ and $19.2 \%$ of perampanel users depending on the study. In comparison, between $4.4 \%$ and $8.3 \%$ of subjects receiving placebo experiences weight gain to this degree. No clear dose-response relationship was identified for weight gain as an adverse event. No laboratory abnormalities or vital sign changes were clearly attributable to perampanel use.

The retrospective monotherapy data included 60 patients in the safety analysis. (p-2) Overall, 12 patients experienced treatment-emergent adverse events, none of which was considered serious. Nine of these events were described. Three patients experienced dizziness, while 2 each experienced balance disorder, depressed mood and irritability. Four withdrawals were reported.

In the study that evaluated perampanel for use in treating tonic-clonic seizures, $82.7 \%$ of subjects receiving active drug experienced treatment-emergent adverse events compared with $72 \%$ who were randomized to placebo. ${ }^{28} \mathrm{Six}$ in each group were deemed severe. There was a single death in each group. The first occurred in the placebo cohort, and was attributed to SUDEP. The second death was a subject in the perampanel group who reportedly drowned. Five subjects in the placebo group and 9 in the active treatment group discontinued therapy due to adverse events. Of these, several had psychiatric events, including 3 placebo subjects ( 2 with suicidal ideation and 1 with agitation, confusion and depression) and 5 perampanel subjects (1 with aggression, and others with anxiety, insomnia, abnormal behavior, mood swings, suicidal ideation and suicide attempt (number of patients not specified)). Non-psychiatric adverse events that were reported by at least $5 \%$ of subjects (placebo vs active treatment) include decreased alertness and altered cognition (14.6\% vs 19.8\%), hostility/aggression (4.9\% vs $18.5 \%)$, accident/injury (11.1\% vs $14.6 \%)$, and rash ( $1.2 \%$ vs $6.2 \%$ ). As with the previous studies, there were no significant abnormalities in laboratory values or vital signs noted with perampanel use.

Additional data has been gleaned from the Phase III studies with regard to specific adverse events. To determine if there were enough laboratory changes in perampanel patients to suggest that the drug might compromise liver function, the data for the trials that enrolled patients with partial seizures was pooled and analyzed. ${ }^{34}$ The total safety population considered consisted of 1,038 subjects receiving perampanel. No doserelated trends were noted when baseline and end-of-treatment values for alkaline phosphatase, alanine aminotransferase, 
aspartate aminotransferase, gamma-glutamyl transferase and bilirubin were compared. Cholelithiasis was noted in 3 subjects taking 4,8 and $12 \mathrm{mg} /$ day, but the connection to treatment was not made.

An additional post hoc analysis of Phase III data as it pertains to falls was also completed for the subjects who were enrolled in the partial seizure trials. ${ }^{35}$ In the active treatment group, $5.1 \%$ of patients fell in comparison with $3.4 \%$ of those receiving placebo. There did appear to be a dose-relationship to the incidence of falling. In addition, those who fell were more likely to be older and had a longer treatment duration than those who did not. No differences were found with regard to the concomitant use of enzyme-inducing antiepileptics, or males vs females. Severity was broken down into mild $(n=32)$, moderate $(n=19)$ or severe $(n=2$ with only one fall believed to be related to high dose [12mg/day] perampanel). Of the falls that occurred, a total of 34 were not believed to be related to perampanel therapy. The fall rate in the clinical trials was similar to the fall rates noted to occur with other medications in the antiepileptic class. ${ }^{36}$

Particular interest has been paid to the psychiatric adverse events identified in patients receiving perampanel therapy. Safety data from Phase I, II and III trials has been analyzed for safety with special concentration on psychiatric events. ${ }^{14}$ Considering Phase III data only, irritability (3\%, 4\%, 7\% and $12 \%$ with placebo, 4,8 and $12 \mathrm{mg}$ /day respectively), and aggression (1\% with placebo and $4 \mathrm{mg} /$ day, $2 \%$ with $8 \mathrm{mg} /$ day and 3\% with $12 \mathrm{mg} /$ day) both appeared to occur in a dose-related fashion. When analyzing the double-blind trials, the open-label trial, and the clinical data derived from non-epileptic patients in earlier trials, the dose-related occurrence of adverse events was also notable for all psychiatric incidents with the exception of sleep disorders. Dose reductions were employed for 8 subjects displaying anger. Two additional patients had treatment discontinued, and did not have resolution of their symptoms at the time of follow-up. Of note, a history or hostility or aggression was not different between the subjects receiving perampanel and those receiving placebo (12.2\% vs $12 \%)$. The subjects without epilepsy that were exposed to drug did not display an increase in the risk of aggression vs those in the placebo group. Display of homicidal ideation occurred in 1 individual in the doubleblind trials, and an additional 4 in the open-label extension trial. It should be noted that adjustments to other medications were allowed in the extension trial making causality harder to prove. There are no systematic reviews of the incidence of homicidal ideation with other antiepileptic agents for comparison. Of note, aggression and irritability have been previously documented in patients receiving levetiracetam, gabapentin, lamotrigine, phenobarbital, tiagabine, topiramate and zonisamide. ${ }^{37}$ In total, $2.5 \%$ of subjects in this analysis stopped treatment due to psychiatric adverse events. Investigators did not employ validated measures for the identification of psychiatric disturbances, nor were the trials designed to do so.

Adverse events from the retrospective study discussed previously also evaluated psychiatric and behavioral adverse events. ${ }^{29}$ In fact, most events reported were psychiatric in nature, including rates of at least $10 \%$ for irritability and aggression. No difference was noted in the rates of adverse events regardless of underlying diagnosis, including when compared with subjects enrolled in the Phase III trials. The authors concluded that psychiatric history should not be a contraindication to perampanel use despite frequent reports of behavioral adverse events post-marketing.

However, other studies suggest that pre-existing behavioral or psychiatric diagnoses are sometimes predictive of adverse events in this domain associated with perampanel use. Data from the previously described retrospective evaluation of patients in the Netherlands with refractory seizures and intellectual disabilities demonstrated a rate of behavioral adverse events of $40.3 \%{ }^{30}$ Those most often reported include agitation, aggression, disruptive behavior and mood alterations. Approximately one-third of patients experienced these events within the first 3 months of therapy initiation, while another $11.9 \%$ reported adverse events after 3 months, but before 6 . Of 19 patients who had previously documented behavioral symptoms associated with their diagnoses, over half had new symptoms in the behavioral domain, or worsening of pre-existing symptoms. In another study that evaluated patient outcomes over 12 months of perampanel use, a history of hyperactivity or personality disorder was associated with a higher likelihood of developing psychiatric adverse events (26.1\% vs $13.7 \%$ for irritability, $7 \%$ vs $3 \%$ for anxiety and $3.2 \%$ vs $0.3 \%$ for psychosis). ${ }^{24}$ In addition, a 2 -year retrospective evaluation of 26 patients in a residential setting who had highly resistant epilepsy in conjunction with some form of cognitive impairment revealed that of 13 who had adverse events, 11 required discontinuation of therapy. ${ }^{38}$ Of those 11,9 were previously diagnosed with psychiatric comorbidity, most often personality and adjustment disorders. The small number of subjects in this report must be taken into consideration. However, notably the authors state that of all antiepileptic medications they employed to treat their patients, perampanel was associated with more psychiatric events than any other. 
Some data indicate that rates of perampanel efficacy in adults and adolescents (without intellectual disability) is similar. ${ }^{39,40}$ However, it has been suggested that behavioral adverse events occur with greater frequency in younger patients. ${ }^{41}$ Whether this is because children with epilepsy often have intellectual disabilities remains to be determined. A retrospective medical record review of children using perampanel has been completed with emphasis on drug tolerability ${ }^{42}$ The mean age for the 24 patients evaluated was 10 years. Four each had been diagnosed with partial (focal) epilepsy and generalized epilepsy, while the remaining 16 carried a diagnosis of both. These were individuals with highly resistant epilepsy as evidenced by a mean number of previous antiepileptics used equaling 8.3 . The mean dose of perampanel that was employed for these patients was $6.6 \mathrm{mg} /$ day. Over a 21 -month time frame, $54 \%$ of patients required discontinuation of perampanel, $62 \%$ of whom ended therapy due to adverse events, with another $15 \%$ claiming both adverse events and lack of efficacy in combination as the reason for stopping treatment. Violent behavior was documented in 3 children, while 2 exhibited psychosis. Individuals aged $>12$ were more likely to experience disturbances of behavior. While none of these patients had been previously diagnosed with a psychiatric disorder, half were categorized as having intellectual disabilities. Another study in patients aged 12-17 years having partial seizures with or without secondary generalization was conducted in part for the evaluation of perampanel on behavior. ${ }^{43}$ Like the Phase III trials, there was a 6-week titration phase followed by 13 weeks of maintenance therapy. One hundred and twenty-three individuals, with an IQ - measured to be at least 70 points, were evaluated. Seventy-nine were taking perampanel and the remainder were given placebo. Aggressive behavior was reported in $8.2 \%$ of individuals on active treatment vs $2.1 \%$ in the placebo group. One of these patients was determined to have symptoms unrelated to perampanel, with 3 each having symptoms designated possibly related or probably related. Of those 7 patients, 2 had a history of psychiatric diagnoses (not specified). Six recovered (3 with dose decrease, and 3 without), while the remaining patient continued to exhibit aggression. None of the patients had their treatment discontinued. It is worth noting that $43.5 \%$ of the perampanel group were also using levetiracetam in comparison with the placebo group in which only $18.8 \%$ were using both therapies concomitantly. No specific explanation for this discrepancy was offered. Currently, there are no data to suggest that the combination of perampanel and levetiracetam produces behavioral or psychiatric adverse events more readily than either drug alone. ${ }^{44}$ While the difference in aggressive behavior was not statistically different between the groups, a trend was noted. Efficacy did not differ regardless of the presence or absence of behavioral disturbance implying that the 2 are not correlated. Utilizing an automated system with task stimuli presented to subjects via notebook computers and requiring responses of "yes" or "no", this same group of subjects was evaluated to see if perampanel had a significant effect on cognitive abilities..$^{45,46}$ Negative outcomes were noted for continuity of attention in perampanel users. However, quality of sporadic memory actually improved in those on perampanel, and while speed of memory declined in both groups, the decrease was slower in the active treatment group. No statistical significance was noted between groups, but additional study of perampanel as a drug that might be associated with memory preservation in patients with epilepsy may be warranted.

\section{Drug interactions}

Antiepileptic inducers of cytochrome $\mathrm{P} 450$ hepatic enzymes have consistently been shown to alter the serum concentrations of perampanel. The impact of inducers appears to be dose-dependant. ${ }^{15}$ Enzyme-inducing antiepileptic drugs (EIAEDs) include carbamazepine, oxcarbazepine, phenytoin, eslicarbazepine, phenobarbital, primidone and topiramate. The most commonly used EIAED in the Phase III trials were carbamazepine and oxcarbazepine $(n=491$ and 270, respectively). ${ }^{47}$ Individuals receiving $12 \mathrm{mg} /$ day doses of perampanel experienced a decrease in serum concentrations with all of the inducers, but to a lesser extent with eslicarbazepine, phenobarbital, primidone and topiramate. In another review completed retrospectively, 160 serum samples that had been collected for therapeutic drug monitoring purposes were analyzed..$^{15}$ The majority of these were collected at random times, usually at least 12 hours after administration due to bedtime dosing. Fifty control subjects not using EIAED were included. The drug found to have the greatest impact on perampanel was carbamazepine, the concomitant use of which resulted in a $69 \%$ decrease in serum concentrations of the former. Oxcarbazepine decreased perampanel concentrations by $37 \%$, topiramate by $18 \%$ and phenytoin by $13 \%$. However, statistical significance for topiramate and phenytoin could not be established due to small sample sizes. The manufacturer has suggested that an increase in perampanel doses may be warranted for individuals taking strong cytochrome $\mathrm{P} 450$ inducers, including non-antiepileptic agents (eg, rifampin and St John's Wort). ${ }^{18}$ However, no specific dosing recommendations have been offered, and no maintenance 
dose for individuals taking EIAED has been established. As such, dosing should be individualized. Of note, the responder rate in the Phase III clinical trials was higher when subjects were not taking EIAED $(p<0.01) .{ }^{23}$ However, the use of these agents occurred more readily in individuals utilizing 3 medications in addition to perampanel, which may be an indication that their epilepsy was more refractory to drug therapy.

Conversely, the effect of perampanel on the clearance of other antiepileptics in the partial seizure trials was evaluated. ${ }^{48}$ The data demonstrated that the clearance of clonazepam, levetiracetam, phenobarbital, phenytoin, topiramate and zonisamide was unaffected by concomitant perampanel. Increased clearance was noted with perampanel $12 \mathrm{mg} /$ day for carbamazepine (4.3\%), clobazam (between 3.4\% and $7.7 \%$ [males vs females]), lamotrigine (9.3\%), and valproic acid (5\%). No clinical significance was noted. However, oxcarbazepine clearance decreased by $26 \%$. Of note, the active metabolite of the drug was not measured. The clinical relevance of this interaction remains unknown. ${ }^{48}$

Interactions that have been documented as occurring with non-antiepileptic medications include decreased serum concentrations of the hormone levonorgestrel (up to 40\%). ${ }^{18}$ The potential effect on the effectiveness of contraceptives containing levonorgestrel as an ingredient is unknown at present, but the use of a backup method of birth control is probably prudent. Midazolam (which is used primarily for its sedative properties, but is sometimes used for the control of seizure activity) has been shown to decrease perampanel concentrations by roughly $14 \%$. The enzyme inhibitor ketoconazole may increase concentrations of perampanel by up to $20 \%$. No specific recommendations are given with regard to these combinations. ${ }^{18}$

\section{Dosage and administration}

Initial treatment with perampanel is done with $2 \mathrm{mg} /$ day at bedtime to minimize the potential for adverse events. ${ }^{18}$ If enzyme inducers are on board, the initial dose is recommended to be increased to $4 \mathrm{mg} /$ day. Increases of $2 \mathrm{mg} /$ day no more often than every week are advised so as to minimize sideeffects. The $12 \mathrm{mg} /$ day dose is the recommended maximum. If a patient has mild hepatic impairment, however, the maximum dose is cut in half to $6 \mathrm{mg} /$ day. For moderate hepatic impairment, the dose is further decreased to $4 \mathrm{mg} / \mathrm{day}$. Bi-weekly titration is recommended for such individuals, as it is for elderly patients as well. No doubling up of doses is recommended for patients who miss one due to the extensive half-life of the drug. However, if therapy is discontinued for at least 3 weeks, it is recommended that the initial dose-titration schedule be employed for therapy re-initiation. ${ }^{49}$

\section{Place in therapy}

Perampanel is a novel, first-in-class antiepileptic agent approved by the FDA for use as monotherapy for partial seizures, and as adjunctive therapy for primarily generalized tonic-clonic seizures. Perampanel's long half-life may make it an ideal choice for an individual who may have issues with adherence. Among the antiepileptics, the drug is generally well tolerated, with dizziness and somnolence being the most common adverse events experienced by users. However, as first came to light in the Phase III clinical studies, psychiatric and behavioral adverse events seem to be more common with perampanel than many other antiepileptic agents. Post-marketing data have demonstrated that both adult and pediatric patients are susceptible to behavioral effects at rates higher than reported in the clinical trials. ${ }^{50}$ Consideration does need to be given to the fact that there is generally a higher incidence of psychiatric and behavioral disorders in patients who have been diagnosed with refractory epilepsy, which may have some effect on the number of patients who report such adverse events when taking perampanel. ${ }^{51}$ These individuals are also more likely to be taking additional medications that have been associated with behavioral changes, including levetiracetam, topiramate, and less frequently, zonisamide. ${ }^{52-54}$ According to a study completed using a hypothetical health plan model of 1 million people, the drug has been estimated to have a per person budget impact of $\$ 0.0055$ after 5 years due to the corresponding decrease in direct and indirect medical costs. ${ }^{55}$ The cost to payers is expected to be minimal, while the cost to society is close to neutral.

\section{Conclusion}

Perampanel is a welcome addition to the antiepileptic drug arsenal. It has consistently been shown to be effective in helping control both partial seizures and generalized tonic--clonic seizures in patients who have failed other therapies.

\section{Disclosure}

The author reports no conflicts of interest in this work.

\section{References}

1. Neligan A, Hauser WA, Sander JW. The epidemiology of the epilepsies. Handb Clin Neurol. 2012;107:113-133.

2. Tolman JA, Faulkner MA. Treatment options for refractory and difficult to treat seizures: focus on vigabatrin. Ther Clin Risk Manag. 2011;7: 367-375.

3. Rheims S, Ryvlin P. Pharmacotherapy for tonic-clonic seizures. Expert Opin Pharmacother. 2014;15:1417-1426.

4. Ben-Menachem E. Medical management of refractory epilepsypractical treatment with novel antiepileptic drugs. Epilepsia. 2014; 55(Suppl 1):3-8. 
5. Plosker GL. Permapanel as adjunctive therapy in patients with partialonset seizures. CNS Drugs. 2012;26(12):1085-1096.

6. Franco V, Crema F, Ludice A, Zaccara G, Grillo E. Novel treatment options for epilepsy: focus on perampanel. Pharmacol Res. 2013;70: 35-40.

7. Glutamate Receptors. In: White HS, Rho JM, eds. Mechanisms of action of antiepileptic drugs. 1st ed. New York: Professional Communications, Inc.; 2010:71-84.

8. Rektor I, Krauss GL, Bar M, et al. Perampanel study 207: long-term open-label evaluation in patients with epilepsy. Acta Neurol Scand. 2012 126(4):263-269.

9. During MJ, Spencer DD. Extracellular hippocampal glutamate and spontaneous seizure in the conscious human brain. Lancet. 1993;341(8861) 1607-1610.

10. Le DA, Lipton SA. Potential and current use of N-metyl-D-aspartate (NMDA) receptor antagonists in diseases of aging. Drugs Aging. 2001; 18(10):717-724.

11. Rogawski MA. Revisiting AMPA receptors as an antiepileptic target. Epilepsy Curr. 2011;11:56-63.

12. Mayer ML. Glutamate receptor ion channels. Curr Opin Neurobiol. 2005; $15: 282-288$.

13. Rosenmund C, Stern-Bach Y, Stevens CF. The tetrameric structure of a glutamate receptor channel. Science. 1998;280:1596-1599.

14. Ettinger AB, LoPresti A, Yang H, et al. Psychiatric and behavioral adverse events in randomized clinical studies of the noncompetitive AMPA receptor antagonist perampanel. Epilepsia. 2015;56(8):1252-1263.

15. Patsalos PN, Gougoulaki M, Sander JW. Perampanel serum concentrations in adults with epilepsy: effect of dose, age, sex and concomitant anti-epileptic drugs. Ther Drug Monit. 2016;38(3):358-364.

16. Laurenza A, Ferry J, Hussein Z. Population pharmacokinetics and pharmacodynamics of perampanel: a pooled analysis from three phase III trials. Epilepsy Curr. 2012;12(Supp1 1):2.231. Abstract.

17. Templeton D. Pharmacokinetics of perampanel, a highly selective AMPAtype glutamate receptor antagonist. Epilepsia. 2009;50(Suppl 11): 98. Abstract.

18. Fycompa (perampanel) package insert. Woodcliff Lake, NJ: Esai, Inc.; 2012.

19. French JA, Krauss GL, Biton V, et al. Adjunctive perampanel for refractory partial-onset seizures: randomized phase III study 304. Neurology. 2012;79(6):589-596.

20. French JA, Krauss GL, Steinhoff BJ, et al. Evaluation of adjunctive perampanel in patients with refractory partial-onset seizures: results of a randomized global phase III study 305. Epilepsia. 2013;54:117-125.

21. Krauss GI, Serratosa JM, Villaneuva V. Adjunctive perampanel for refractory partial-onset seizures: randomized phase III study 306. Neurology. 2012;78(18):1408-1415.

22. Montouris G, Yang H, Williams B, Zhou S, Laurenza A, Fain R. Efficacy and safety of perampanel in patients with drug-resistant partial seizures after conversion from double-blind placebo to open-label perampanel. Epilepsy Res. 2015;114:131-140.

23. Glauser T, Laurenza A, Haichen Y, Williams B, Ma T, Fain R. impact of adjunct perampanel based on number of antiepileptic drugs at baseline and baseline predictors of efficacy: a phase III post-hoc analysis. Epilepsy Res. 2016;119:34-40.

24. Villanueva V, Garcés M, López-González, et al. Safety, efficacy and outcome-related factors of perampanel over 12 months in a real-world setting: the FYDATA study. Epilepsy Res. 2016;126:201-210.

25. FDA approves Eisai's FYCOMPA ${ }^{\circledR}$ (perampanel) for Use as Monotherapy for the Treatment of Partial-Onset Seizures [press release] Woodcliff Lake, NJ. Eisai Co., Ltd; 2017. Available from: http://eisai. mediaroom.com/2017-07-26-FDA-Approves-Eisais-FYCOMPA-Rperampanel-for-Use-as-Monotherapy-for-the-Treatment-of-PartialOnset-Seizures. Accessed September 7, 2017.

26. Kwan P, Gil-Nagel A, Laurenza A, Patten A, Cartwright K. Evaluation of perampanel monotherapy in epilepsy: prospective open-label extension and retrospective uncontrolled studies. Poster presented at: 69th Annual Meeting of the American Academy of Neurology; April 22-28, 2017; Boston, MA.
27. Gil-Nagel A, Burd S, Toledo M, et al. A retrospective, multicenter study to investigate dosage, efficacy and safety of perampanel given as monotherapy in routine clinical care in people with epilepsy. Poster presented at: 12th European Congress on Epileptology; September 11-15, 2016; Prague, Czech Republic.

28. French JA, Krauss GI, Wechsler RT, et al. Perampanel for tonic-clonic seizures in idiopathic generalized epilepsy. Neurology. 2015;85:950-957.

29. Maurousset A, Limousin N, Praline J, Biberon J, Corcia P, De toffol B. Adjunctive perampanel in refractory epilepsy: experience at tertiary epilepsy care center in Tours. Epilepsy Behav. 2016;61:237-241.

30. Snoeijen-Schouwenaars FM, van Ool JS, Tan IY, Schelhaas HJ, Majoie MH. Evaluation of perampanel in patients with intellectual disability and epilepsy. Epilepsy Behav. 2017;66:64-67.

31. Leppik IE, Wechsler RT, Williams B, Yang H, Zhou S, Laurenza A. Efficacy and safety of perampanel in the subgroup of elderly patients included in the phase III epilepsy clinical trials. Epilepsy Res. 2015; 110:216-220.

32. Krauss GL, Bar M, Biton V, et al. Tolerability and safety of perampanel: two randomized dose-escalation studies. Acta Neurol Scand. 2012; 125:8-15.

33. Krauss GL, Perucca E, Ben-Menachem E, et al. Perampanel, a selective, noncompetitive $\alpha$-amino-3-hydroxy-5-methyl-4-isoxazolepropionic acid receptor antagonist, as adjunctive therapy for refractory partialonset seizures: interim results from phase III, extension study 307 . Epilepsia. 2013;54:126-134.

34. Laurenza A, Yang H, Williams B, Zhou S, Ferry J. Absence of liver toxicity in perampanel-treated subjects: pooled results from partial seizures phase III perampanel clinical studies. Epilepsy Res. 2015;113:76-85.

35. Leppik IE, Yang H, Williams B, et al. Analysis of falls in patients with epilepsy enrolled in the perampanel phase III randomized double-blind studies. Epilepsia. 2017;58:51-59.

36. Shiek AB, Hill KD, O'Brien TJ, et al. Falls and fractures in patients chronically treated with antiepileptic drugs. Neurology. 2012;79:145-151.

37. Piedad J, Rickards H, Besag FM, et al. Beneficial and adverse psychotropic effects of antiepileptic drugs in patients with epilepsy: a summary of prevalence, underlying mechanisms and data limitations. CNS Drugs. 2012;26:319-335.

38. Huber B, Schmid G. A two-year retrospective evaluation of perampanel in patients with highly drug-resistant epilepsy and cognitive impairment. Epilepsy Behav. 2017;66:74-79.

39. Rosenfeld W, Rozentals G, Yang H, et al. Efficacy and safety of adjunctive perampanel in the subgroup of adolescent patients with refractory, partial onset seizures included in the 3 double-blind, placebo-controlled phase III clinical trials. 41st Annual Meeting of the Child Neurology Society; 31 October-3 November; 2012; Huntington Beach, AC, USA.

40. Conry J, Lagae L, Yang H, et al. Interim analysis of efficacy and safety of adjunctive perampanel in the adolescent population in the open-label extension phase of the double-blind, placebo-controlled, parallel-group studies in patients with refractory partial onset seizures. 41st Annual Meeting of the Child Neurology Society; 31 October-3 November; 2012; Huntington Beach, AC, USA.

41. Renfroe B, Yang H, Williams B. Interim efficacy and safety analysis of adjunctive perampanel in the adolescent population from the extension phase of 3 double-blind placebo-controlled phase 3 (core) studies in patients with refractory partial-onset seizures. 42nd Annual Meeting of the Chile Neurology Society; 30 October-2 November; 2013; Austin, TX, USA.

42. Heyman E, Lahat E, Levin N. Tolerability and efficacy of perampanel in children with refractory epilepsy. Dev Med Child Neurol. 2017;59: 441-444.

43. Lagae L, Villanueva V, Meador KJ, et al. Adjunctive perampanel in adolescents with inadequately controlled partial-onset seizures: a randomized study evaluating behavior, efficacy, and safety. Epilepsia. 2016;57(7): $1120-1129$

44. Trinka E, Steinhoff BJ, Nikanorova M, Brodie MJ. Perampanel for focal epilepsy: insights from early clinical experience. Acta Neurol Scand. 2016;133:160-172. 
45. Wesnes KA, Busner J. Differential effects on attention over 24 weeks of an NMDA antagonist vs carbamazepine in pediatric epilepsy patients. Eur Neurophsychopharmacol. 2013;23:S602.

46. Meador KJ, Yang H, Piňa-Garza JE, Laurenza A, Kumar D, Wesnes KA. Cognitive effects of adjunctive perampanel for partial-onset seizures: a randomized trial. Epilepsia. 2016;57:243-251.

47. Kwan P, Brodie MJ, Laurenza A, FitzGibbon H, Gidal BE. Analysis of pooled phase III trials of adjunctive perampanel for epilepsy: impact of mechanism of action and pharmacokinetics on clinical outcomes. Epilepsy Res. 2015;117:117-124.

48. Majid O, Laurenza A, Ferry J, Hussein Z. Impact of perampanel on pharmacokinetics of concomitant antiepileptics in patients with partial-onset seizures: pooled analysis of clinical trials. Br J Clin Pharmacol. 2016; $82: 422-430$.

49. Greenwood J, Valdes J. Perampanel (Fycompa). A review of clinical efficacy and safety in epilepsy. Drug Forecast. 2016;41:683-688.

50. Rubboli G. Perampanel in refractory epilepsies: what real-life experience tells us. Dev Med Child Neurol. 2017;59:352-353.
51. Lin JJ, Mula M, Hermann BP. Uncovering the neurobehavioural comorbidities of epilepsy over the lifespan. Lancet. 2012;380:1180-1192.

52. Cramer JA, De Rue K, Devinsky O, et al. A systematic review of the behavioral effects of levetiracetam in adults with epilepsy, cognitive disorders, or an anxiety disorder during clinical trials. Epilepsy Behav. 2003; 4:124-132.

53. Mula M, Trimble MR, Lhatoo SD, et al. Topiramate and psychiatric adverse events in patients with epilepsy. Epilepsia. 2003;44:659-663.

54. Stephen LJ, Kelly K, Parker P, et al. Levetiracetam monotherapyoutcomes from an epilepsy clinic. Seizure. 2011;20:554-557.

55. Tremblay G, Barghout V, Patel V, Tsong W, Wang Z. Budget impact of perampanel as adjunctive treatment of uncontrolled partial-onset and primary generalized tonic-clonic seizures in the United States. Epilepsy and Behav. 2017;68:196-202.
Drug Design, Development and Therapy

\section{Publish your work in this journal}

Drug Design, Development and Therapy is an international, peerreviewed open-access journal that spans the spectrum of drug design and development through to clinical applications. Clinical outcomes, patient safety, and programs for the development and effective, safe, and sustained use of medicines are the features of the journal, which

\section{Dovepress}

has also been accepted for indexing on PubMed Central. The manuscript management system is completely online and includes a very quick and fair peer-review system, which is all easy to use. Visit http://www.dovepress.com/testimonials.php to read real quotes from published authors.

Submit your manuscript here: http://www.dovepress.com/drug-design-development-and-therapy-journal 